\title{
Diacronie
}

Studi di Storia Contemporanea

$\mathrm{N}^{\circ} 35,3 \mid 2018$

Gli strumenti di Clio

\section{Giorgio Sacchetti, Vite di partito. Traiettorie esistenziali nel PCI togliattiano. Priamo Bigiandi (1900-1961)}

\section{Carmelo Albanese}

\section{(2) OpenEdition \\ Journals}

Edizione digitale

URL: http://journals.openedition.org/diacronie/9628

DOI: $10.4000 /$ diacronie. 9628

ISSN: 2038-0925

Editore

Association culturelle Diacronie

Notizia bibliografica digitale

Carmelo Albanese, «Giorgio Sacchetti, Vite di partito. Traiettorie esistenziali nel PCI togliattiano. Priamo Bigiandi (1900-1961) », Diacronie [Online], № 35, 3 | 2018, documento 27, Messo online il 29 septembre 2018, consultato il 23 septembre 2020. URL : http://journals.openedition.org/diacronie/9628; DOI : https://doi.org/10.4000/diacronie.9628 


\title{
Diacronie
}

Studi di Storia Contemporanea

\section{$35,3 / 2018$}

Gli strumenti di Clio: uomini, luoghi e teorie della storia dalla tradizione critica alla comunicazione digitale

\section{RECENSIONE: Giorgio SACCHETTI, Vite di partito. Traiettorie esistenziali nel PCI togliattiano. Priamo Bigiandi (1900-1961), Napoli, Edizioni Scientifiche Italiane, 2016, 200 pp.}

\author{
A cura di Carmelo ALBANESE
}

Per citare questo articolo:

ALBANESE, Carmelo, «RECENSIONE: Giorgio SACCHETTI, Vite di partito. Traiettorie esistenziali nel PCI togliattiano. Priamo Bigiandi (1900-1961), Napoli, Edizioni Scientifiche Italiane, 2016, 200 pp.», Diacronie. Studi di Storia Contemporanea : Gli strumenti di Clio: uomini, luoghi e teorie della storia dalla tradizione critica alla comunicazione digitale, 35, 3/2018, 29/09/2018,

URL: < http://www.studistorici.com/2018/09/29/albanese_numero_35-2/ >

Diacronie Studi di Storia Contemporanea $\rightarrow$ http://www.diacronie.it

Rivista storica online. Uscita trimestrale.

redazione.diacronie@hotmail.it

Comitato di direzione: Naor Ben-Yehoyada - João Fábio Bertonha - Christopher Denis-Delacour - Maximiliano Fuentes Codera Anders Granås Kjøstvedt - John Paul Newman - Deborah Paci - Niccolò Pianciola - Spyridon Ploumidis - Wilko Graf Von Hardenberg

Comitato di redazione: Jacopo Bassi - Luca Bufarale - Gianluca Canè - Luca G. Manenti - Fausto Pietrancosta - Alessandro Salvador - Matteo Tomasoni - Luca Zuccolo

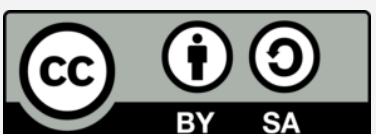

Diritti: gli articoli di Diacronie. Studi di Storia Contemporanea sono pubblicati sotto licenza Creative Commons 3.0. Possono essere riprodotti e modificati a patto di indicare eventuali modifiche dei contenuti, di riconoscere la paternità dell'opera e di condividerla allo stesso modo. La citazione di estratti è comunque sempre autorizzata, nei limiti previsti dalla legge. 


\title{
27/ RECENSIONE: Giorgio SACCHETTI, Vite di partito. Traiettorie esistenziali nel PCI togliattiano. Priamo Bigiandi (1900-1961), Napoli, Edizioni Scientifiche Italiane, 2016, 200 pp.
}

\author{
A cura di Carmelo ALBANESE
}

Giorgio Sacchetti, docente a contratto di Storia delle ideologie del Novecento in Europa presso l'Università di Padova, è uno studioso del movimento operaio, in particolare del territorio aretino, con una attenzione privilegiata alla tendenza anarchica ${ }^{1}$. In quest'ambito, oltre a svariate pubblicazioni volte a ricostruire genesi e forme del conflitto sociale e politico nel Valdarno ${ }^{2}$, ha tratteggiato i profili biografici di diversi suoi protagonisti ${ }^{3}$. Dopo un primo lavoro sull'anarchico Otello Gaggi ${ }^{4}$, si è occupato del percorso politico ed esistenziale di Priamo Bigiandi, minatore antifascista di Cavriglia (Arezzo) e, dopo la Liberazione, sindaco del suo paese, consigliere provinciale e deputato comunista al Parlamento per due legislature, di cui il volume esaminato costituisce la seconda edizione - con diverso titolo - aggiornata ${ }^{5}$, ed è compendiato dalla raccolta di alcune testimonianze di Pietro Amendola, Orazio Barbieri, Enzo Brogi (già sindaco di Cavriglia), Mauro Ferri e della figlia di Bigiandi, Alba, raccolte tra il 1997 e il $1998^{6}$. Trattandosi di una ricostruzione biografica, la ricerca copre un arco cronologico ampio, ma è certamente il periodo 1948-1958, ossia quello in cui il dirigente comunista svolge il mandato parlamentare, che ne costituisce il fulcro. Fonti principali della pubblicazione (articolata in sei capitoli) sono le carte dell'Archivio privato della famiglia Bigiandi (5 buste e una cartella), la documentazione di polizia

\footnotetext{
${ }^{1}$ SACCHETTI, Giorgio, Carte di gabinetto. Gli anarchici italiani nelle fonti di polizia (1921-1991), Ragusa, La Fiaccola, 2015.

${ }^{2}$ ID., «Sindacalisti e anarchici: il socialismo rivoluzionario valdarnese e aretino ai primi del Novecento», in Annali Aretini, IV, 1996, pp. 179-191; ID., Ligniti per la Patria. Collaborazione, conflittualità, compromesso. Le relazioni sindacali nelle miniere del Valdarno superiore (1915-1958), Roma, Ediesse, 2002; ID., Sovversivi e squadristi. 1921: alle origini della guerra civile in provincia di Arezzo, Roma, Aracne, 2010.

${ }^{3}$ Vedi le voci da lui curate in ANTONIOLI, Maurizio, BERTI, Giampietro, FEDELE, Santi, IUSO, Pasquale (a cura di), Dizionario biografico degli anarchici italiani, 2 voll., Pisa, BFS, 2003-2004.

${ }^{4}$ SACCHETTI, Giorgio, Otello Gaggi. Vittima del fascismo e dello stalinismo, Pisa, BFS, 1992.

${ }^{5}$ ID., Il minatore deputato. Priamo Bigiandi (1900-1961), Firenze, Manent, 1998.

${ }^{6}$ ID., Vite di partito. Traiettorie esistenziali nel PCI togliattiano. Priamo Bigiandi (1900-1961), Napoli, Edizioni Scientifiche Italiane, 2016, pp. 173-190.
} 
e dell'Archivio della Federazione comunista di Arezzo e gli articoli coevi di alcuni organi di stampa locali.

La struttura tematica del libro è funzionale all'obiettivo dell'autore di affrontare questioni che vanno al di là della vicenda del deputato di Cavriglia - presto emarginato «dalla macchina implacabile del Partito» per «miserevoli ragioni (come invidie, gelosie e interessi locali di infimo cabotaggio) $»^{7}-$, ovvero dare conto dei «meccanismi autoritari e dirigistici che sovrintendevano alla gestione degli affari locali e della formazione dei quadri di una federazione locale del PCI negli anni Cinquanta» ${ }^{8}$ : un sistema che, a detta dell'autore, si riproduce ancora alla fine degli anni Novanta, condizionando persino le vicende della committenza e della pubblicazione della prima edizione del volume, quando, ormai sciolto il PCI, pure «rimanevano quegli atteggiamenti clientelari e di spocchia autoreferenziale, quel controllo, fastidioso pervasivo e tracotante, negli assessorati e nelle istituzioni culturali accademiche e di ricerca governate dai postcomunisti» ${ }^{9}$. A fronte di tali proponimenti, l'impianto complessivo del volume suscita più di una perplessità, innanzitutto per ciò che attiene alla caratura e all'opera effettivamente svolta dal protagonista, certo personaggio politico di rilievo sul piano locale, ma nelle cui vicissitudini si ha difficoltà ad intravedere, come invece vorrebbe l'autore, tratti emblematici di una storia più grande e complessa. Tali ostacoli emergono chiaramente man mano che si scorre la ricostruzione biografica, che più di una volta disattende le aspettative suscitate dalle altisonanti titolazioni dei capitoli. Nel primo di questi, ad esempio, «La sovversione sociale come scuola di vita», si introduce la genesi dell'antifascismo nel Valdarno a partire dai tragici "fatti" del marzo 1921 ove, negli scontri tra operai delle miniere di Castelnuovo dei Sabbioni, fascisti e forza pubblica, e in sommosse successive, due persone perdono la vita. Nel processo, che inizia due anni dopo, Bigiandi figura tra i settantacinque imputati e viene condannato per correità in omicidio e altri delitti minori a undici anni di reclusione (poi ridotti a nove e mezzo). Esce di prigione nel maggio 1927, a seguito di indulto, dopo sei anni di reclusione. In realtà, non sappiamo se effettivamente egli abbia svolto un ruolo nei tragici eventi, visto che nelle diverse udienze le testimonianze sembrano contraddirsi e che l'imputato durante l'interrogatorio afferma «di essersi trovato in casa il giorno della sommossa»" ${ }^{10}$ sta di fatto che quelli del Valdarno sono gli unici "fatti" riportati dall'autore ascrivibili, in qualche modo, alla categoria di "sovversione" e, a parte alcune testimonianze di contatti, non vi sono altri cenni nel volume che alludono ad una qualche iniziativa dell'operaio aretino in grado di dare conto della intestazione del capitolo.

\footnotetext{
${ }^{7}$ Ibidem, pp. 7-8.

${ }^{8}$ Ibidem, p. 9.

${ }^{9}$ Ibidem.

${ }^{10}$ Ibidem, pp. 26-27.
} 
Il profilo politico di Bigiandi si costruisce effettivamente nel secondo dopoguerra in relazione alle problematiche del bacino lignitifero. Eletto sindaco di Cavriglia nella primavera del 1946 nelle liste del PCI, si trova a gestire la spinosa questione delle risorse comunali che, a causa della crisi mineraria e della distruzione da parte dei tedeschi della centrale termica, si sono ridotte di oltre un terzo per l'esaurimento degli introiti prima derivanti dalla presenza nel territorio delle Società mineraria ed elettrica. La cronica difficoltà del settore estrattivo del Valdarno nella competizione di mercato e la salvaguardia dei lavoratori sono al centro dell'attività amministrativa di Bigiandi (che termina nel 1951, mentre prosegue quella di consigliere provinciale) e, soprattutto, di quella parlamentare $^{11}$. Su questo terreno, a partire dal 1956, emerge la diversità di vedute con il PCI aretino che appoggia il progetto governativo per potenziare l'escavazione a cielo aperto mediante macchine a grande potenziale e per costruire un grande complesso industriale che preveda l'utilizzo in loco della lignite (cap. 5). Da questo tornante - nello sviluppo economico dell'area e del percorso politico di Bigiandi («Più dell’Ungheria, più del XX congresso del PCUS, è il Cinquantasei valdarnese la vera rivoluzione») $)^{12}$ - muove l'autore per svolgere le proprie valutazioni sull'esecrabile modus operandi del PCI togliattiano e sulla condotta dei suoi dirigenti locali, per i quali il mito sovietico rappresenterebbe una «immagine salvifica» e indiscutibile e in cui, come dimostrerebbe il caso di Bigiandi, anche in presenza di un dissidio «prevale [...] un senso di adesione alla disciplina di partito, intesa come postulato di fede e appartenenza a un "chiesa" in cui il dirigente deve assolvere alla funzione di "sacerdozio". Per questo l'obbedienza è un imperativo, eresia il dissenso»" ${ }^{13}$.

Tali giudizi non sembra siano supportati da un approfondito confronto con l'indagine storiografica, eccezion fatta per il lavoro di sintesi di Franco Andreucci ${ }^{14}$, definito sulle pagine di questa rivista «un esperimento alquanto ben riuscito di "Public History", tanto nell'accezione peggiorativa di distorsione, quanto in quella neutra di divulgazione» ${ }^{15}$. Eppure, come a ragione ha rilevato lo studioso testé menzionato, «la storiografia sul PCI è stata a lungo uno dei maggiori campi di ricerca della storiografia italiana in generale, paragonabile, per le sue proporzioni, almeno fino agli anni Ottanta, agli studi storici sul fascismo» ${ }^{16}$. Una mole di ricerche e contributi immensa che, anche nella diversità di posizioni e letture, ha messo abbondantemente in luce la

\footnotetext{
${ }^{11}$ In generale, la grande maggioranza delle proposte di legge presentate in Parlamento nelle prime legislature repubblicane riguarda questioni locali e micro-interessi socio-economici. Cfr. MORISI, Massimo, Le leggi del consenso. Partiti e interessi nei primi parlamenti della Repubblica, Soveria Mannelli, Rubbettino, 1992.

${ }^{12}$ SACCHETTI, Giorgio, Vite di partito, cit., p. 123.

${ }^{13}$ Ibidem, p. 137.

${ }^{14}$ ANDREUCCI, Franco, Da Gramsci a Occhetto. Nobiltà e miseria del Pci 1921-1991, Pisa, Della Porta Editori, 2014.

${ }^{15}$ DI GIACOMO, Michelangela, «RECENSIONE: Franco ANDREUCCI, Da Gramsci a Occhetto. Nobiltà e miseria del PCI 1921-1991, Pisa, Della Porta Editori, 2014, 467 pp.», in Diacronie. Studi di Storia Contemporanea : Le dittature militari: fisionomia ed eredità politica, 24, 4/2015, URL:

<http://www.studistorici.com/2015/12/29/digiacomo_numero_24/> [consultato il 30 agosto 2018].

${ }^{16}$ ANDREUCCI, Franco, op. cit., p. 12.
} 
complessità della articolazione organizzativa comunista e delle "vite di partito"17, evidenziando, per un verso, come il mito dell'URSS, più che rappresentare una icona sacrale, abbia costituito innanzitutto un potente fattore di mobilitazione nella costruzione del partito comunista più grande d'Europa $^{18}$, e, per un altro, che lo stesso ruolo dei vertici del partito italiano - e di Togliatti in primis - nel rapporto con il partito sovietico fosse tutt'altro che riducibile a quello di mero esecutore di ordini ${ }^{19}$. Sorprende, dunque, l'assenza di riferimenti ad una tale copiosa letteratura: non tanto, è bene ribadirlo, rispetto alla ricostruzione biografica in sé, quanto in relazione a quel complesso universo a cui l'autore intende approdare.

Un aspetto sul quale il lavoro di Sacchetti fornisce elementi di conoscenza originali riguarda la Federazione di Arezzo che, similmente a quelle di Pisa e di Lucca, entro un quadro regionale dell'organizzazione comunista nel secondo dopoguerra estremamente differenziato - sul piano della penetrazione tra le masse contadine e operaie, come anche del rapporto tra partito e sindacato -, si caratterizza per una scarsa propensione a recepire - e contribuire a far affermare la strategia della "democrazia progressiva", spingendo i vertici nazionali ad intervenire con un lungo commissariamento ${ }^{20}$. Come rilevato in una importante ricerca del 1981 sul Partito comunista in Toscana, in questa provincia si determina nell'immediato dopoguerra una doppia frattura - che solo nel tempo, «anche attraverso la contrastata conquista di un'egemonia sul piano elettorale», avrà modo di sanarsi - che contrappone centro e periferia (con una netta prevalenza della seconda, ossia della provincia operaia e mezzadrile) e, soprattutto, la "vecchia

\footnotetext{
${ }^{17}$ A titolo puramente esemplificativo cfr. ILARDI, Massimo, ACCORNERO, Aris (a cura di), Annali della Fondazione Feltrinelli : Il partito comunista italiano. Struttura e storia dell'organizzazione 1921-1979, XXI, 1981; MARTINELlI, Renzo, Storia del Partito comunista italiano, 2 voll., Torino, Einaudi, 1995-1998, che, pur collocandosi in continuazione con la celebre Storia di Paolo Spriano (SPRIANO, Paolo, Storia del Partito comunista italiano, 5 voll., Torino, Einaudi, 1967-1975), ne aggiorna l'impianto, non limitandosi a tracciare la storia dei gruppi dirigenti comunisti ma allargando lo sguardo a tutto il partito, sia sul versante della struttura che su quello della caratterizzazione socio-antropologica del suo apparato e della sua base. Cfr. SANTOMASSIMO, Gianpasquale, «Note e discussioni. La nuova storia del Pci», in Italia Contemporanea, 205, 1996, pp. 713-721, in particolare pp. 716 et seq.

${ }^{18}$ Come sottolineato da Giovanni Gozzini, al rientro in Italia di Togliatti, nel 1944, il PCI era «un mostrum» di due milioni di iscritti: cifra, questa, enormemente più alta sia della $\mathrm{DC}$ che del partito sovietico (che contava tre milioni di iscritti su 170 milioni di abitanti) e degli altri paesi europei. «Tra gli anni quaranta e cinquanta - scrive ancora lo storico - quello che Duverger definisce il "tasso di adesione", vale a dire il rapporto percentuale tra iscritti ed elettori, oscilla tra l'otto e il nove dei partiti socialisti francese e tedesco e tra il cinque e il sei dei laburisti, mentre per i comunisti italiani oltrepassa il trenta per cento». Cfr. GOZZINI, Giovanni, «Partito comunista e storia d'Italia», in Italia Contemporanea, 194, 1994, pp. 180-186, la citazione è a p. 184 .

${ }^{19}$ Cfr., ad esempio, FLORES, Marcello, GALLERANO, Nicola, Sul Pci. Un'interpretazione storica, Bologna, Il Mulino, 1992; TINÈ, Salvatore, HÖBEL, Alexander (a cura di), Palmiro Togliatti e il comunismo del Novecento, Roma, Carocci, 2016; GOZZINI, Giovanni, Il primato della politica estera: Togliatti e la democrazia italiana, in HÖBEL, Alexander (a cura di), Togliatti e la democrazia italiana, Roma, Editori Riuniti, 2017, pp. 203-229.

${ }^{20}$ Cfr. ROSSI, Mario Giuseppe, SANTOMASSIMO, Gianpasquale, Il Partito Comunista Italiano: introduzione, in ROTELLI, Ettore (a cura di), La ricostruzione in Toscana dal CLN ai partiti, vol. II, I partiti politici, Bologna, Il Mulino, 1981, pp. 757-768, in particolare p. 766.
} 
guardia" di estrazione operaia e i giovani (prevalentemente intellettuali) iscritti dal 1944-1945 ${ }^{21}$. Nella difficoltà di affermazione di un gruppo dirigente autoctono riconosciuto, l'attività del partito si cristallizza in una scrupolosa ossessione "organizzativista" che sovrasta - fin quasi ad annullarle - le riflessioni e i confronti di ordine più propriamente politico, mentre la vita interna al partito è contraddistinta da beghe, rivalità e personalismi tipici delle circoscritte realtà comunali ma che, proprio per tale ragione, contraddice la definizione dell'autore di «partitosagrestia» ${ }^{22}$.

Più che ad una presunta invadenza degli organismi di direzione nazionali, dunque, è a questo contesto, di cui anche Bigiandi è attore e protagonista, che va ricondotta la vicenda legata alla esclusione - sua e del senatore uscente Galliano Gervasi - dalle liste per le elezioni nazionali del $1958^{23}$ : una scelta ufficialmente motivata dal fatto che il parlamentare ha già svolto due mandati e che il partito intende "portare nuove forze in parlamento ${ }^{24}$, ma in ogni caso decisa in una riunione congiunta del Comitato Federale e della Commissione di Controllo nella quale solo quattro dei quarantadue dirigenti presenti si esprimono in suo favore ${ }^{25}$. L'impegno politico di Bigiandi prosegue ancora nel Consiglio provinciale, dove viene rieletto nell'autunno del 1960, ma, gravemente ammalato, muore il 20 agosto 1961.

\footnotetext{
${ }^{21}$ GASPARRI, Tamara, MARTINELLI, Renzo, Il Partito Comunista Italiano: appunti per una ricerca, in ROTELLI, Ettore (a cura di), op. cit., pp. 769-980, soprattutto pp. 894-903, la citazione è a p. 903.

${ }^{22} \mathrm{Ci}$ si riferisce al titolo del sesto capitolo, «Federazioni di provincia: un partito-sagrestia». SACCHETTI, Giorgio, Vite di partito, cit., pp. 133-171.

${ }^{23}$ In merito alla influenza delle dinamiche politiche locali sulla declinazione territoriale delle culture politiche, importanti e originali suggestioni sono contenute nel numero monografico della rivista Meridiana intitolato Fare politica, 90, 2017.

${ }^{24}$ È quanto afferma Catone Maestrelli, della segretaria del partito, all'assemblea della sezione "Gherardi" del 17 febbraio 1958. SACCHETTI, Giorgio, Vite di partito, cit., p. 144.

${ }^{25}$ Verbale della riunione del C.F. e della C. Federale di controllo del 10 marzo 1958, cit. in ibidem, p. 149.
} 


\section{L'AUTORE}

Carmelo ALBANESE (1979) ha conseguito il titolo di dottore di ricerca in Storia contemporanea all'Università di Firenze con una tesi sulla lotta politica in Sicilia in età giolittiana ( $\mathrm{I}^{\circ}$ Premio "Spadolini Nuova Antologia" - XVIII edizione). Interessato ai temi dell'antifascismo, della Resistenza e della società italiana fra le due guerre, dal 2014 collabora con l'Istituto Storico Toscano della Resistenza e dell'Età Contemporanea (ISRT). Tra le sue recenti pubblicazioni si segnalano i volumi Leto Fratini, scultore. Percorsi esistenziali e traiettorie dell'antifascismo tra Firenze e Milano (Pisa, Pacini Editore, 2017) e Storia dell'Anpi Oltrarno. Associazionismo popolare e memoria della Resistenza a Firenze (Firenze, Anpi Oltrarno, 2017).

URL: < http://www.studistorici.com/progett/autori/\#Albanese $>$ 